ORIGINALES

\title{
Impulsividad funcional y disfuncional en jóvenes con consumo intensivo de alcohol (binge drinking)
}

\section{Functional and dysfunctional impulsivity in young binge drinkers}

AnA AdAN

Departamento de Psiquiatría y Psicobiología Clínica. Facultad de Psicología. Universidad de Barcelona.

Instituto de Investigación en Cerebro, Cognición y Conducta (IR3C).

\section{Resumen}

El patrón de consumo intensivo de alcohol (CIA), especialmente practicado por jóvenes, se ha convertido en un problema social y de salud importante. A pesar de ello, sólo en los últimos años se está delimitando su prevalencia, las consecuencias a corto y medio plazo y los posibles factores de riego. El presente trabajo se propone estudiar, por primera vez, las posibles diferencias en las dimensiones de impulsividad funcional y disfuncional del modelo de Dickman entre estudiantes universitarios con CIA y no consumidores de alcohol o controles. Participaron 80 sujetos con patrón de CIA (40 hombres) y 80 controles (40 hombres), de edades entre los 18 y 25 años $(21,38 \pm 1,91)$, los cuales respondieron al Inventario de Impulsividad de Dickman (DII, 1990). Se controlaron diversas variables que se conoce pueden influir en los resultados, entre ellas la tipología circadiana. Las puntuaciones de impulsividad disfuncional fueron superiores en el grupo CIA en comparación con el control, mientras que no se observaron diferencias en la impulsividad funcional. Los hombres aportaron mayor nivel de impulsividad tanto funcional como disfuncional que las mujeres, aunque en esta última ello es significativo sólo en el grupo CIA. La utilización del DII se ha mostrado sensible al CIA, pudiendo ser una herramienta de evaluación a incorporar en abordajes preventivos y/o de tratamiento en el futuro.

Palabras clave: alcohol, consumo intensivo alcohol, impulsividad funcional, impulsividad disfuncional, personalidad, sexo.
Enviar correspondencia a:

Ana Adan

Departamento de Psiquiatría y Psicobiología Clínica.

Facultad de Psicología. Universidad de Barcelona.

Passeig Vall d'Hebrón 171. 08035 Barcelona.

Correo-e: aadan@ub.edu

\section{Abstract}

The pattern of binge drinking (BD), found especially among young people, has become an important social and health problem. However, its prevalence has not been carefully studied until recently, together with its consequences in the mid and long run and the possible risk factors. The aim of this work is to study, for the first time, the possible differences in the dimensions of functional and dysfunctional impulsivity in Dickman's model among university students with $\mathrm{BD}$ and non-consumers of alcohol or control group. 80 subjects ( 40 men) with a pattern of BD and 80 controls (40 men) participated in the study, aged 18 to $25(21,38 \pm 1,91)$. They all completed Dickman's Impulsivity Inventory (DII, 1990). Several variables known to affect the results, such as circadian typology, were controlled for. The scores on dysfunctional impulsivity were higher in the BD group compared to the controls, while no differences were found according to functional impulsivity. Men showed a higher degree of impulsivity than women, both functional and dysfunctional, although in dysfunctional impulsivity this is significant only in the BD group. The DII has proven sensitive to detecting BD, and thus it could become an assessment tool to take into account in preventive or treatment approaches in the future.

Key words: alcohol, binge drinking, functional impulsivity, dysfunctional impulsivity, personality, sex. 
E n los últimos años ha emergido un patrón de consumo de alcohol intermitente, en forma de atracones, concentrado en sesiones de pocas horas, asociado principalmente a las noches de fin de semana y que se realiza con grupos de iguales. Este patrón, denominado consumo intensivo de alcohol (binge drinking o heavy episodic drinking), se caracteriza por picos de incidencia entre los jóvenes, igualación del consumo entre sexos, rejuvenecimiento del perfil de los bebedores abusivos y escasa percepción de riesgo (Parada, Corral, Caamaño-Isorna, Mota, Crego, Rodríguez-Olguín et al., 2011 para una revisión).

Los estudios epidemiológicos aportan datos muy variables respecto a la prevalencia del consumo intensivo de alcohol (CIA), pero las estadísticas de los últimos informes en España presentan datos preocupantes. Así, la última encuesta domiciliaria sobre alcohol y drogas del Observatorio Español sobre Drogas (2010) ha puesto de manifiesto un aumento del $\mathrm{CIA}$, que considerando los últimos 30 días es del $28,4 \%$ en hombres y del 17,9\% en mujeres de 15 a 24 años. El CIA en jóvenes se asocia de forma consistente con policonsumo, entre el que destaca el cannabis con alrededor de un 50\% de prevalencia (Cadaveira, 2009) y es un factor de riesgo para el desarrollo posterior de abuso/dependencia de alcohol (Balodis, Potenza y Olmstead, 2009; Perry y Carroll, 2008; White, Marmorstein, Crews, Bates, Mun y Loeber, 2011). El estudio del posible impacto cognitivo del $\mathrm{CIA}$ pone en evidencia déficits en el rendimiento neuropsicológico similares a los observados en bebedores crónicos (Field, Schoenmakers y Wiers, 2008; Garcia-Moreno, Expósito, Sanhueza y Alguno et al., 2008; Scaife y Duka, 2009), en especial en tareas que implican actividad prefrontal. La evaluación de la toma de decisiones mediante la lowa Gambling Task, en concreto, ha mostrado el patrón de "miopía" para las consecuencias futuras, similar al de los pacientes con adicción y que supone una conducta impulsiva con menor control inhibitorio (Johnson, Xiao, Palmer, Sun, Wang, Wei et al., 2008), de mayor impacto en los hombres (Goudriaan, Grekin y Sher, 2011). Stephens y Duka (2008), en su revisión, concluyen que el CIA produce alteraciones funcionales de hiperactividad en la corteza prefrontal y la amígala, las cuales pueden comportar excitotoxicidad.

La impulsividad, considerada uno de los principales rasgos de personalidad, ha centrado el interés de numerosos trabajos en la última década por hallarse relacionada con numerosos trastornos mentales incluidos en el DSM-IV-R y diversas conductas desadaptativas (Guerrieri, Nederkoorn y Janse, 2008; Paaver, Eensoo, Pulver y Harro, 2006; VigilColet, Morales-Vives y Tous, 2008). Ésta se define como la tendencia a actuar con falta de previsión, sin evaluar las consecuencias que puedan derivarse. Cabe destacar que es una de las variables más consistentes relacionadas con el contacto temprano a las drogas, la repetición de los consumos y la progresión a la adicción, así como una condición que se exacerba con el consumo (Pedrero, 2009; Perry y Carrol, 2008; Verdejo, Lawrence y Clark, 2008) y promueve la recaída (Cano, Araque y Ortiz, 2011). En jóvenes con patrón CIA también se ha observado elevada impulsividad, en estudios recientes (Field et al., 2008; White et al., 2011). La impulsividad puede ser la clave en la peor ejecución de tareas ejecutivas tradicionalmente observada en pacientes con trastorno adictivo (Dolan, Bechara y Nathan, 2008), así como en adolescentes (Castellanos-Ryan, Rubia y Conrod, 2011).

Si bien la mayoría de los cuestionarios de personalidad incluyen una dimensión de impulsividad, se han desarrollado instrumentos específicos para su medición desde distintos marcos teóricos como la escala de impulsividad de Barratt (BIS 11) y el cuestionario de Impulsividad-AventurerismosEmpatía creado por Eysenck (IVE). Entre ellos se halla el Inventario de Impulsividad de Dickman (DII, Dickman, 1990), basado en el modelo de impulsividad propuesto por el mismo autor el cual hace hincapié en que las consecuencias de la impulsividad no son siempre negativas. Propone la existencia de dos tipos de impulsividad, la funcional relacionada con la tendencia a tomar decisiones rápidas cuando así lo requiere una situación en beneficio de la persona y la disfuncional relacionada con decisiones rápidas e irreflexivas que comportan consecuencias negativas para la persona. Tanto los cuestionarios de personalidad como los especificos de impulsividad consideran en sus evaluaciones sólo la dimensión disfuncional.

Se han observado diferencias entre sexos en las puntuaciones de impulsividad funcional (Adan, Natale, Caci y Prat, 2010; Caci, Nadalet, Baylé, Robert y Boyer, 2003; Claes, Vertommen y Braspenning, 2000) y, en muestras de población general, también en la impulsividad disfuncional (Adan et al., 2010; Claes et al., 2000). En ambos casos, los hombres presentan puntuaciones superiores. Ello es congruente con la constatación de que los hombres tienen menor autocontrol y un riesgo incrementado de sufrir alteraciones de los impulsos respecto a las mujeres (Maccallum, Blaszczynski, Ladouceur y Nover, 2007; Paaver et al., 2006; Strüber, Lück y Roth, 2008; Verdejo et al., 2008), siendo ello más evidente cuando se estudian jóvenes (Goodriaan et al, 2011; Perry y Carroll, 2008). Puntuaciones elevadas en impulsividad disfuncional se asocian a cogniciones desadaptativas (Mobini, Grant, Kass y Yeomans., 2007) y déficits en tareas de inhibición conductual y funciones ejecutivas (Vigil-Colet, 2007; Vigil-Colet et al., 2008). El DIl se ha mostrado sensible a trastornos adictivos y del control de los impulsos (Meerkerk, van den Eijnden, Franken y Garretsen, 2010; Pedrero, 2009), hallándose puntuaciones de impulsividad disfuncional superiores en las muestras clínicas. Sin embargo, ningún trabajo precedente ha estudiado si existe relación entre las dos dimensiones de impulsividad de DIl y el patrón de CIA en jóvenes.

El presente trabajo explora, por primera vez, las posibles diferencias entre estudiantes universitarios con patrón de $\mathrm{CIA}$ y no consumidores de alcohol en las dos dimensiones de impulsividad del modelo de Dickman. Asímismo, se estudia si el sexo puede ser una variable moduladora en los resultados observados. 


\section{Método}

\section{Participantes}

Los participantes fueron 160 estudiantes universitarios de psicología de la Universidad de Barcelona, de edad comprendida entre los 18 y 25 años $(21,38 \pm 1,91)$. De ellos, 80 cumplian criterios de CIA (40 hombres) y 80 que no consumían alcohol esporádicamente en grandes cantidades (40 hombres) constituyeron el grupo control. No existían diferencias de edad entre hombres $(21,36 \pm 1,97)$ y mujeres $(21,39 \pm 1,86)\left[\mathrm{t}_{(1,138)}=0,083 ; p=0,934\right]$ ni entre el grupo de CIA $(21,32 \pm 1,71)$ y el control $(21,42 \pm 2,10)\left[\mathrm{t}_{(1,138)}=\right.$ $0,330 ; p=0,742]$.

Mediante una entrevista de inclusión estructurada se desestimaron aquellos sujetos con consumo diario de drogas, exceptuando la nicotina y cafeína a dosis moderadas. Otros criterios de exclusión fueron la dependencia al alcohol u otras drogas, historia familiar de alcoholismo, así como antecedentes o presencia actual de enfermedades médicas o psiquiátricas. Los sujetos del grupo control no eran consumidores esporádicos de elevadas cantidades de alcohol, manifestando como máximo un consumo ocasional de dos bebidas de baja graduación (en especial cerveza) al mes. El patrón de CIA se consideró de al menos 5 consumiciones alcohólicas en hombres y de 4 en mujeres tomadas en un intervalo de 2 horas, al menos una vez en los últimos 30 días (NIAAA, 2004; Parada et al., 2011). Este consumo se producía mayoritariamente asociado a dias de fin de semana, aunque algunos de ellos lo efectuaban los jueves por la noche, día que muchos jóvenes salen en grupo en la actualidad. Del grupo CIA se excluyeron sujetos con consumo esporádico reiterado de otras drogas (cannabis, cocaína, etc.). Finalmente, se controló la tipología circadiana (matutinos, intermedios o ningún tipo y vespertinos) por ser un factor que se relaciona tanto con diferencias en rasgos de personalidad como con el consumo de drogas (Adan, 2010; Prat y Adan, 2011), seleccionándose sólo participantes de tipología intermedia. La tipología matutina se ha configurado como un factor protector de elevada impulsividad disfuncional y del consumo de drogas. Así mismo, la afectación de la ritmicidad circadiana con el consumo de drogas es un marcador temprano en el riesgo de aparición de patologías físicas y psíquicas (Adan y Sánchez-Turet, 1995; 2000).

Todos los sujetos participaron de forma voluntaria, no retribuida y tras firmar su consentimiento informado a la inclusión en el estudio. Los datos que se presentan en este trabajo pertenecen a un estudio más amplio sobre factores de riesgo y repercusiones neuropsicológicas del consumo intensivo de alcohol en jóvenes. Los participantes fueron entrevistados individualmente y respondieron una batería de cuestionarios, entre los que se hallaban el DII de impulsividad y el CSM de tipología circadiana, junto a información de variables demográficas. Sólo los participantes que completaron los cuestionarios se consideraron en los análisis. La comisión ética de la Universidad de Barcelona aprobó el protocolo del estudio y éste cumplía con los principios de la declaración de Helsinki.

\section{Instrumentos de evaluación}

El Inventario de Impulsividad de Dickman (DII; Dickman, 1990) consta de 23 items, 11 de los cuales evalúan la impulsividad funcional (ej. "Me gustaria realizar un trabajo que requiriera tomar decisiones rápidas") y 12 la impulsividad disfuncional (ej. "Hago y digo cosas sin tener en cuenta sus consecuencias"). Hemos utilizado la versión española del DII (Chico, Tous, Lorenzo-Seva y Vigil-Colet, 2003) con el formato de respuesta modificado en lugar de dicotómico (si/no) con una escala Likert de 5 puntos considerándose 1 (totalmente en desacuerdo) y 5 (totalmente en acuerdo). El rango de puntuación para la impulsividad funcional es de 11 a 55 y el de la disfuncional de 12 a 60 . El cuestionario con este formato de respuesta ha mostrado propiedades psicométricas adecuadas en la muestra no clínica de mayor tamaño estudiada hasta el momento (Adan et al., 2010), con una consistencia interna de 0,759 para la impulsividad funcional y de 0,801 para la disfuncional. El DII muestra una buena validez convergente en su formato original de respuesta (Caci et al., 2003; Chico et al., 2003; Claes et al., 2000; Pedrero, 2009).

Para controlar la tipología circadiana se utilizó la versión española de la escala compuesta de matutinidad (CSM; Adan, Caci y Prat, 2005). La escala se halla constituida por 13 items cuya puntuación oscila entre 0 y 55 y la asignación de una tipología intermedia se corresponde con puntuaciones de 26 a 36. La escala aporta excelentes propiedades psicométricas, con una consistencia interna de 0,870 en población española, y validez transcultural (Caci, Adan, Bohle, Natale, Pornpitakpan y Tilley, 2005).

\section{Análisis estadístico}

Se realizaron análisis descriptivos y la fiabilidad interna del DIl se estimó mediante el coeficiente $\alpha$ de Cronbach. Se calcularon correlaciones de Pearson entre las puntuaciones de impulsividad funcional y disfuncional, así como entre éstas y la edad. Se llevó a cabo un análisis múltiple de la covarianza (MANCOVA) considerando las puntuaciones de las dos dimensiones de impulsividad del DII como variables dependientes, el consumo de alcohol ( $\mathrm{CIA} /$ control) y el sexo como variables independientes y la edad como covariable para controlar sus posibles efectos. Se calculó el estadístico Eta al cuadrado parcial $\left(\eta_{\mathrm{p}} 2\right)$ para medir el tamaño de los efectos y la potencia estadística observada de los efectos principales fue superior a 0,700 en todos los casos. Los análisis se han efectuado con el paquete estadístico SPSS (versión 15.0) y los tests estadísticos son bilaterales con un error tipo I establecido en el 5\%.

\section{Resultados}

La Tabla 1 presenta el rango, media y error estándar de las dos dimensiones de impulsividad para el total de la muestra, los grupos consumo de alcohol y sexo. La consistencia interna ( $\alpha$ de Cronbach) de ambas dimensiones de impulsividad fue adecuada y superior al criterio mínimo aceptable 
$(0,70)$, tanto considerando el total de los participantes del estudio como los distintos grupos (Tabla 1). El análisis correlacional mostró asociaciones positivas significativas entre la impulsividad funcional y disfuncional considerando todos los sujetos $(r=0,312 ; p<0,01)$ y ambos grupos de consumo, si bien la correlación fue superior en el grupo de CIA ( $r$ $=0,361 ; p<0,01)$ que en el control $(r=0,283 ; p<0,05)$. Mientras que en el grupo de mujeres también se observó una asociación positiva entre ambas dimensiones de impulsividad $(r=0,344 ; p<0,01)$, en el de hombres no alcanzó el nivel de significación $(r=0,202)$. Así mismo, la edad aportó una correlación positiva significativa sólo con las puntuaciones de impulsividad funcional $(r=0,286 ; p<0,01)$, lo que sustenta considerarla como covariable en los análisis para asegurar que no influya en los resultados obtenidos.

Tabla 1. Estadísticos descriptivos (rango, media \pm error estándar) y coeficiente $\alpha$ de Cronbach para las puntuaciones del Inventario de Impulsividad de Dickman (IID) en la muestra total y los grupos de consumo de alcohol y sexo.

\begin{tabular}{|c|c|c|c|c|c|}
\hline \multirow[b]{2}{*}{ IID } & \multirow[b]{2}{*}{$\begin{array}{c}\text { Total } \\
(\mathrm{N}=160)\end{array}$} & \multicolumn{2}{|c|}{ Consumo de alcohol } & \multicolumn{2}{|c|}{ Sexo } \\
\hline & & $\begin{array}{c}\mathrm{CIA} \\
(\mathrm{N}=80)\end{array}$ & $\begin{array}{l}\text { Control } \\
(\mathrm{N}=80)\end{array}$ & $\begin{array}{l}\text { Hombres } \\
(\mathrm{N}=80)\end{array}$ & $\begin{array}{l}\text { Mujeres } \\
(N=80)\end{array}$ \\
\hline \multirow{3}{*}{ IF } & $12-49$ & $12-46$ & $19-49$ & $20-49$ & $12-48$ \\
\hline & $32,32 \pm 0,51$ & $32,77 \pm 0,68$ & $31,86 \pm 0,72$ & $33,51 \pm 0,66$ & $31,13 \pm 0,76$ \\
\hline & $\alpha=0,747$ & $\alpha=0,781$ & $\alpha=0,729$ & $\alpha=0,769$ & $\alpha=0,721$ \\
\hline \multirow{3}{*}{ ID } & $13-49$ & $14-49$ & $13-45$ & $13-49$ & $13-48$ \\
\hline & $30,31 \pm 0,58$ & $33,78 \pm 0,68$ & $26,84 \pm 0,65$ & $32,13 \pm 0,68$ & $28,49 \pm 0,72$ \\
\hline & $\alpha=0,816$ & $\alpha=0,821$ & $\alpha=0,960$ & $\alpha=0,808$ & $\alpha=0,986$ \\
\hline
\end{tabular}

Notas. $\mathrm{CI} \mid \mathrm{A}=$ Consumo Intensivo de $\mathrm{Alcohol} ; \mathrm{IF}=\mathrm{Impul}$ sividad Funcional; $\mathrm{ID}=\mathrm{Impulsividad}$ Disfuncional .

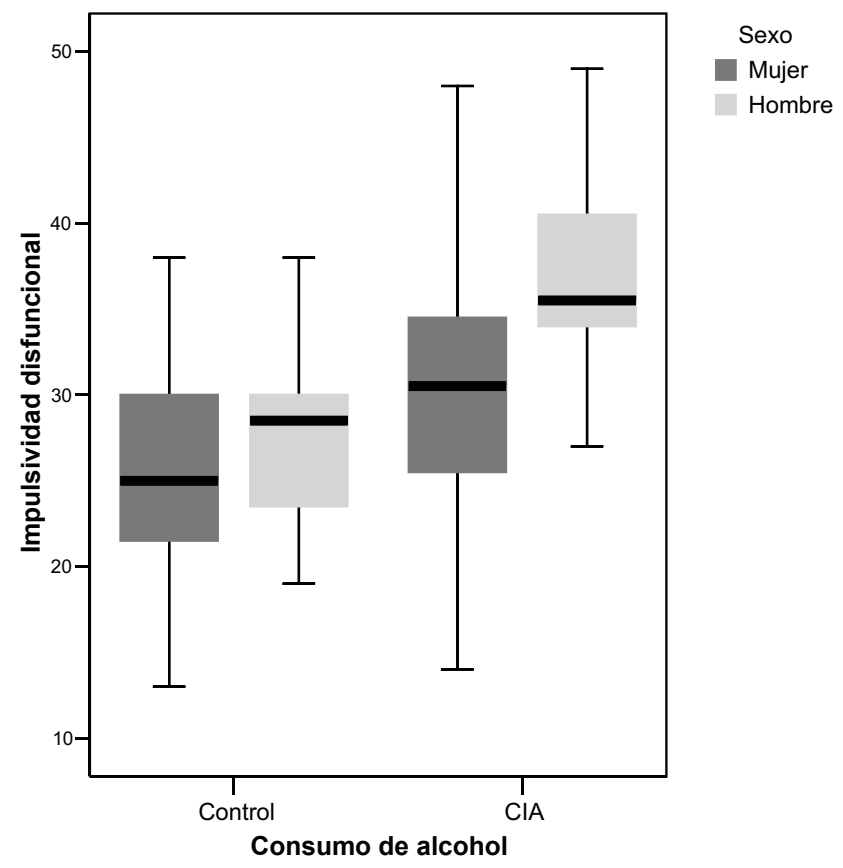

Figura 1. Diagrama de cajas de la interacción entre las variables consumo de alcohol y sexo para las puntuaciones de impulsividad disfuncional del Inventario de Impulsividad de Dickman. El rango de puntuación es de 12 a 60 . CIA: consumo intensivo de alcohol.
EI MANCOVA aportó diferencias significativas entre grupos de consumo sólo para la impulsividad disfuncional $\left(F_{(1,155)}\right.$ $\left.=49,53 ; p<0,0001 ; \eta_{p} 2=0,242\right)$. El grupo de CIA presenta puntuaciones superiores respecto al grupo control. También la variable sexo mostró efectos significativos, tanto para la impulsividad funcional $\left(F_{(1,155)}=5,92 ; p<0,016 ; \eta_{p} 2=0,037\right)$ como para la disfuncional $\left(F_{(1,155)}=13,52 ; p<0,0001 ; \eta_{p} 2=\right.$ 0,080 ). En ambos casos los hombres son quienes presentan puntuaciones superiores. Ver Tabla 1 para los descriptores de los distintos grupos (media \pm error estándar).

Se obtuvo un efecto significativo para la interacción entre el consumo de alcohol y el sexo en las puntuaciones de impulsividad disfuncional $\left(F_{(1,155)}=4,64 ; p<0,033 ; \eta_{p} 2=\right.$ $0,029$; potencia observada $=0,571)$. La superioridad en las puntuaciones de los hombres respecto a las mujeres se produce sólo en el grupo $\mathrm{CI}$, mientras que en el control aunque los hombres aportan una puntuación media mayor ésta no alcanza diferencias significativas (Figura 1).

\section{Discusión}

Nuestro trabajo es el primero que ha explorado las posibles diferencias de impulsividad funcional y disfuncional, utilizando el modelo propuesto por Dickman (1990), entre jóvenes con patrón de CIA y controles. Los datos apuntan que el DII es adecuado para la evaluación de la impulsividad en el CIA y sugieren su utilidad en la investigación futura en este ámbito. Además, se ha tenido en cuenta el sexo de los participantes y se han controlado numerosas variables que podrian haber influido en nuestras observaciones, entre las que destaca la tipología circadiana, lo que confiere mayor robustez a los resultados.

Se confirman las diferencias de sexo en la impulsividad, con una superioridad de los hombres respecto a las mujeres tanto en la dimensión funcional (Adan et al., 2010; Caci et al., 2003; Claes et al., 2000) como en la disfuncional (Adan, 2010; Claes et al., 2000). Ello se observa también utilizando otros instrumentos de evaluación de la impulsividad y converge con numerosas evidencias procedentes tanto de población normal como de muestras clínicas (Balodis et al., 2009; Field et al., 2008; Perry y Carroll, 2008). Se ha sugerido que la mayor impulsividad de los hombres puede relacionarse con un menor funcionamiento del sistema neurotransmisor serotoninérgico (Paaver et al., 2006; Pattij y Vanderschuren, 2008; Verdejo et al., 2008), siendo ello también decisivo para las diferencias entre sexos existentes en el control de impulsos (Strüber et al., 2008).

La impulsividad disfuncional se considera un marcador de vulnerabilidad para el desarrollo de trastornos adictivos (Paaver et al., 2006; Pedrero, 2009; Verdejo et al., 2008), juego patológico y otras adicciones conductuales (Maccalum et al., 2007; Meerkerk et al., 2010), así como conducta agresiva y antisocial (Strüber et al., 2008; Vigil-Colet et al., 2008). Nuestro trabajo permite afirmar que la dimensión de impulsividad disfuncional del modelo de Dickman también se muestra sensible al fenómeno del $\mathrm{CIA}$, a diferencia de otros cuestionarios de impulsividad que no han consegui- 
do detectar diferencias entre CIA y controles (Balodis et al., 2009; Castellanos-Ryan et al., 2011). Aunque deben llevarse a cabo más estudios con mayor muestra a la utilizada en el presente trabajo, la impulsividad disfuncional quizás en el futuro pueda considerarse un marcador de vulnerabilidad para el CIA.

De gran interés es la interacción obtenida entre el CIA y el sexo para la dimensión de impulsividad disfuncional. Ya que la mayor impulsividad observada en los hombres respecto a las mujeres en el total de la muestra viene determinada por el grupo con CIA. La presencia de puntuaciones elevadas de impulsividad disfuncional puede ser un factor de riesgo para el $\mathrm{CIA}$ y el posterior progreso hacia trastornos de consumo de alcohol y otras drogas, en especial en los hombres. Balodis et al. (2009) hallaron diferencias entre hombres y mujeres con $\mathrm{CIA}$ en el número de bebidas consumidas y expectativas de beber, siendo las de las mujeres de mejorar su sociabilidad y capacidad sexual mientras que la de los hombres era reducir la tensión, observando que ambas variables se hallaban relacionadas con la impulsividad. Tener en cuenta el sexo puede ser esencial en el estudio del CIA, ya que parecen existir especificidades de sexo cruciales de delimitar tanto a nivel básico como clínico.

Se debe evaluar si la impulsividad es un factor mediador en otros aspectos asociados al $\mathrm{CIA}$, en especial el deterioro neuropsicológicos de las funciones ejecutivas que implican toma de decisiones e inhibición (García-Moreno et al., 2008; Johnson et al., 2008; Scaife y Duka, 2009; Stephens y Duka, 2008). Puede que, tal y como observaron Dolan et al. (2008) en pacientes adictos, la consideración de los niveles de impulsividad minimice o haga desaparecer las diferencias de rendimiento neuropsicológico. En este sentido, Goudriaan et al. (2011) han observado que la peor toma de decisiones de sujetos con CIA se produce sólo en el caso de los hombres, quienes nos han aportado niveles superiores de impulsividad. El DII puede resultar una herramienta útil y quizás mejor que otros cuestionarios específicos de impulsividad para ello. El estudio genético de Colzato, van der Wildenberg, van der Does y Hommel (2010) obtuvo que una elevada impulsividad disfuncional se asocia a un polimorfismo del gen DRD2 dopaminérgico relacionado con dificultades conductuales de producir respuestas inhibitorias, mientras que el BIS no mostró relación. Así, se nos abren hipótesis alternativas para avanzar en el conocimiento del impacto del CIA que deberán ser exploradas convenientemente.

Nuestro trabajo presenta algunas limitaciones a superar en futuras investigaciones. Los resultados no pueden extrapolarse a los individuos con CIA en general, ya que sólo se han estudiado estudiantes universitarios de un rango de edad limitado. Sin embargo, los estudiantes son una población diana en el estudio del CIA ya que muestran una prevalencia superior respecto a la de los jóvenes no estudiantes (Balodis et al., 2009; Slutske, 2005). No hemos incluido mediciones relacionadas con el patrón de consumo más allá del criterio CIA ni de ejecución objetiva de funciones frontales y capacidad de inhibición, por lo que no se ha podido explorar el papel predictor de la impulsividad disfuncional en ellas. Sin embargo, los datos obtenidos abundan en la importancia de la impulsividad en el CIA, como White et al. (2011) también han concluido al observar que ésta se asocia a un patrón $\mathrm{CIA}$ más intensificado. Además la elevada impulsividad se relaciona con mayores tasas de recaída en pacientes drogodependientes (Cano et al., 2011) y peor respuesta al tratamiento en jugadores patológicos (Maccallum et al., 2007). Por ello, no cabe duda del beneficio que puede suponer el incorporar aspectos que mejoren la capacidad de respuestas inhibitorias, tanto en programas de prevención como de intervención de consumo de drogas y otros problemas conductuales en la adolescencia y la juventud temprana

\section{Agradecimientos}

Este trabajo ha contado con una subvención por parte del Ministerio de Ciencia e Innovación Español (proyecto PSI2009-12300, subprograma PSIC) y de la Delegación del Gobierno para el Plan Nacional sobre Drogas (BOE 306, 23/12/2003).

\section{Conflicto de intereses}

La autora del artículo manifiesta no tener ningún conflicto de intereses.

\section{Referencias}

Adan, A. (2010). Ritmicidad circadiana y adicción. Editorial. Adicciones, 22, 5-9.

Adan, A., Caci, H. y Prat, G. (2005). Reliability of the Spanish version of the Composite Scale of Morningness. European Psychiatry, 20, 503-509.

Adan, A., Natale, V., Caci, H. y Prat, G. (2010). Relationship between circadian typology and functional and dysfunctional impulsivity. Chronobiology International, 27, 606-619.

Adan, A. y Sánchez-Turet, M. (1995). Smoking effects on diurnal variations of cardiovascular parameters. International Journal of Psychophysiology, 20, 189-198.

Adan, A. y Sánchez-Turet, M. (2000). Effects of smoking on diurnal variations of subjective activation and mood. Human Psychopharmacology Clinical and Experimental, 15, 287-293.

Balodis, I.M., Potenza, M.N. y Olmstead, M.C. (2009). Binge drinking in undergraduates: relationships with sex, drinking behaviours, impulsivity, and the perceived effects of alcohol. Behavioral Pharmacology, 20, 518-526.

Caci, H., Adan, A., Bohle, P., Natale, V., Pornpitakpan, C. y Tilley, A.J. (2005). Transcultural properties of the Composite Scale of Morningness: the relevance of the "morning affect" factor. Chronobiology International, 22, 523-540.

Caci, H., Nadalet, L., Baylé, F.J., Robert, P. y Boyer, P. (2003). Functional and dysfunctional impulsivity: contribution to the construct validity. Acta Psychiatrica Scandinavica, 107, 34-40.

Cadaveira, F. (2009). Alcohol y cerebro adolescente. Adicciones, 21, 9-14.

Cano, G.J., Araque, F. y Ortiz, A.C. (2011). Adicción, impulsividad y curvas temporales de deseo. Adicciones, 23, 141-148. 
Castellanos-Ryan, N., Rubia, K. y Conrod, P.J. (2011). Response inhibition and reward response bias mediate the predictive relationships between impulsivity and sensation seeking and common and unique variance in conduct disorder and substance misuse. Alcoholism: Clinical and Experimental Research, 35, 140155.

Chico, E., Tous, J.M., Lorenzo-Seva, U. y Vigil-Colet, A. (2003). Spanish adaptation of Dickman's impulsivity inventory: its relationship to Eysenck's personality questionnaire. Personality and Individual Differences, 35, 1883-1892.

Claes, L., Vertommen, H. y Braspenning, N. (2000). Psychometric properties of the Dickman Impulsivity Inventory. Personality and Individual Differences, 29, 27-35.

Colzato, L.S., van der Wildenberg, W.P.N., van der Does, A.J.W. y Hommel, B. (20010). Genetic markers of strital dopamine predict individual differences in dysfunctional, but not functional impulsivity. Neuroscience, 170, 782-788.

Dickman, S.J. (1990). Functional and dysfunctional impulsivity: personality and cognitive correlates. Journal of Personality and Social Psychology, 58, 95-102.

Dolan, S.L., Bechara, A. y Nathan, P.E. (2009). Executive dysfunction as a risk marker for substance abuse: the role of impulsive personality traits. Behavioral Sciences and the Law, 26, 799-822.

Field, M., Schoenmakers, T. y Wiers, R.W. (2008). Cognitive processes in alcohol binges: a review and research agenda. Current Drug Abuse Reviews, 1, 263-279.

Garcia-Moreno, L.M., Expósito, J., Sanhueza, C. y Alguno, M.T. (2008). Actividad prefrontal y alcoholismo de fin de semana en jóvenes. Adicciones, 20, 271-280.

Goudriaan, A.E., Grekin, E.R. y Sher, K.J. (2011). Decision making and response inhibition as predictor of heavy alcohol use: a prospective study. Alcoholism: Clinical and Experimental Research, $35,1-8$.

Guerrieri, R., Nederkoorn, C. y Janse, A. (2008). The effect of an impulsive personality on overeating, and obesity: current state of affairs. Psychological Topics 17, 265-286.

Johnson, C.A., Xiao, L., Palmer, P., Sun, P., Wang, O., Wei, Y., Jia, Y., Grenard, J.L., Stacy, A.W. y Bechara, A. (2008). Affective decisionmaking deficits, linked to dysfunctional ventromedial prefrontal cortex, revealed in 10th grade Chinese adolescent binge drinkers. Neuropsychologia, 46, 714-726.

Maccallum, F., Blaszczynski, A., Ladouceur, R. y Nover, L. (2007). Functional and dysfunctional impulsivity in pathological gambling. Personality and Individual Differences, 43, 1829-1838.

Meerkerk, G.J., van den Eijnden, R.J.J.M., Franken, I.H.A y Garretsen, H.F.L. (2010). Is compulsive internet use related to sensitivity to reward and punishment, and impulsivity? Computers in Human Behavior, 26, 729-735.

Mobini, S., Grant, A., Kass, A.E. y Yeomans, M.R. (2007). Relationships between functional and dysfunctional impulsivity, delay discounting and cognitive distortions. Personality and Individual Differences, 43, 1117-1128.

National Institute on Alcohol Abuse and Alcoholism. (2004). NIAAA council approves definition of binge drinking. NIAAA Newsletter, №3. Winter.

Observatorio Español sobre Drogas. Delegación del Gobierno para el Plan Nacional sobre Drogas (2010). Encuesta domiciliaria sobre alcohol y drogas de España (EDADES). 2009/2010.
Disponible en: www.msps.es/gabinetePrensa/notaPrensa/.../ presentacionEdades200910.ppt

Paaver, M., Eensoo, D., Pulver, A. y Harro, J. (2006). Adaptative and maladaptative impulsivity, platelet monoamine oxidase (MAO) activity and risk-admitting in different types of risky drivers. Psychopharmacology, 186, 32-40.

Parada, M., Corral, M., Caamaño-Isorna, F., Mota, N., Crego, A., Rodríguez-Olguín, S. y Cadaveira, F. (2011). Definición del concepto de consumo intensivo de alcohol adolescente (binge drinking). Adicciones, 23, 53-63.

Pattij T. y Vanderschuren, JMJ. (2008). The neuropharmacology of impulsive behaviour. Trends in Pharmacological Sciences, 29, 192199.

Pedrero, E.J. (2009). Evaluación de la impulsividad funcional y disfuncional en adictos a sustancias mediante el inventario de Dickman. Psicothema, 21, 585-591.

Perry, J.L. y Carroll, M.E. (2008). The role of impulsive behavior in drug abuse. Psychopharmacology, 200, 1-26.

Prat, G. y Adan, A. (2011). Influence of circadian typology on drug consume, hazardous alcohol use and hangover symptoms. Chronobiology International, 28, 248-257.

Scaife, J.C. y Duka, T. (2009). Behavioural measures of frontal lobe function in a population of young social drinkers with binge drinking pattern. Pharmacology, Biochemistry and Behavior, 93, 354-362.

Slutske, W.S. (2005). Alcohol use disorders among US college students and their non-college-attending peers. Archives of General Psychiatry, 62, 321-327.

Stephens, D.N. y Duka, T. (2008). Cognitive and emotional consequences of binge drinking: role of amygdala and prefrontal cortex. Philosophical Transactions of the Royal Society of Biological Sciences, 363, 3169-3179.

Strüber, D., Lück, M. y Roth, J. (2008). Sex, aggression and impulse control: an integrative account. Neurocase 14:93-121.

Verdejo, A., Lawrence, A.J. y Clark, L. (2008). Impulsivity as a vulnerability marker for substance-use disorders: review of findings from high-risk research, problem gamblers and genetic association studies. Neuroscience \& Biobehavioral Reviews, 32, 777-810.

Vigil-Colet, A. (2007). Impulsivity and decision making in the balloon analogue risk-taking task. Personality and Individual Differences, 43, 37-45.

Vigil-Colet, A., Morales-Vives, F. y Tous, J. (2008). The relationships between functional and dysfunctional impulsivity and aggression across different samples. Spanish Journal of Psychology, 11, 480487.

White, H.R., Marmorstein, N.R., Crews, F.T., Bates, M.E., Mun, E-Y. y Loeber, R. (2011). Associations between heavy episodic drinking and changes in impulsive behavior among adolescent boys. Alcoholism: Clinical and Experimental Research, 35, 295-303. 